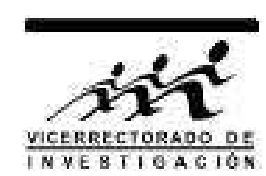

\title{
Estudio de la influencia de la temperatura y el tiempo de tratamiento térmico sobre la formación del cuasicristal i- $\mathrm{Al}_{64} \mathrm{Cu}_{23} \mathbf{F e}_{13}$
}

\author{
M. Z. Pinto*1,2, M. Pillaca ${ }^{1}$ y C. V. Landauro ${ }^{1}$ \\ ${ }^{1}$ Facultad de Ciencias Físicas, Universidad Nacional Mayor de San Marcos, Lima, Perú \\ ${ }^{2}$ Facultad de Ciencias Químicas, Físicas y Matemáticas, Universidad Nacional San Antonio Abad del \\ Cusco, Cusco, Perú
}

Recibido 8 marzo 2012 - Aceptado 15 mayo 2012

\begin{abstract}
En el presente trabajo se estudia la influencia de la temperatura y el tiempo de tratamiento térmico sobre la estabilidad del cuasicristal $\mathrm{Al}_{64} \mathrm{Cu}_{23} \mathrm{Fe}_{13}$. Para ello, las aleaciones de $\mathrm{AlCuFe}$, sintetizadas empleando el método de horno de arco, fueron sometidas a tratamientos térmicos variando dos parámetros: el tiempo y la temperatura.

La caracterización estructural de las muestras obtenidas se realizó mediante la difracción de rayos X y la microscopía electrónica de barrido, mientras que la composición elemental se determinó empleando la técnica de energía dispersiva de rayos X.

Los resultados indican que el tratamiento térmico es indispensable para eliminar fases espurias que están siempre presentes luego del proceso de sintetización de la aleación. También se ha encontrado que tratamientos térmicos de $800{ }^{\circ} \mathrm{C}$ durante 48 horas o $700{ }^{\circ} \mathrm{C}$ durante 168 horas son los más adecuados para obtener muestras con los mayores tamaños de grano $(\sim 90 \mathrm{~nm})$. Finalmente, el análisis elemental indica que durante la síntesis de la aleación se produce una ligera variación de la composición ideal.

Palabras claves: Cuasicristales, tratamiento térmico, difracción de rayos X.
\end{abstract}

\section{Study of the influence of the temperature and the heat treatment time on the formation of the $\mathrm{i}-\mathrm{Al}_{64} \mathrm{Cu}_{23} \mathrm{Fe}_{13}$ quasicrystral}

The influence of the temperature and the heat treatment time on the $\mathrm{Al}_{64} \mathrm{Cu}_{23} \mathrm{Fe}_{13}$ quasicrystal stability is studied in the present work. For this purpose the samples obtained by arc-furnace undergo different heat treatments varying the time and temperature of treatment. The structural characterization of the samples were carried out employing X-ray diffraction, and scanning electron microscopy, whereas the elemental composition was determined using energy dispersive x-ray spectroscopy.

The results indicate that heat treatments are necessary to eliminate spurious phases which are always present after the synthesis of the samples. We also found that heat treatments of $800{ }^{\circ} \mathrm{C}$ by 48 hours or $700{ }^{\circ} \mathrm{C}$ by 168 hours are the most convenient ways to obtain samples with larger grain sizes $(\sim 90$ $\mathrm{nm}$ ). Finally, the elemental analysis indicates a light deviation of the ideal composition due to the synthesis procedure.

Keywords: Quasicrystals, heat treatment, X-ray diffraction.

Tradicionalmente, la estructura atómica de los sólidos puros se dividía en dos clases: las fases cristalinas, estructura ordenada de forma periódica, y las fases amorfas, estructuras desordenadas [1. La primera posee simetrías de traslación y de rotación, orden de corto y de largo alcance, respectivamente, mientras que la segunda sólo posee orden local, de corto alcance. Sin embargo, en 1984 Shechtman y colaboradores reporta- ron [2] una forma intermedia del estado sólido, entre cristales y amorfos, llamada cuasicristal; éstos sistemas presentan una estructura ordenada a largo alcance pero de forma aperiódica, sin simetría de traslación [2]. Además, los cuasicristales son materiales que presentan propiedades físicas sumamente interesantes en comparación con sus componentes por separado; como por ejemplo, alta resistencia eléctrica a temperatura am-

*milidazarella@gmail.com 
biente [3, bajo coeficiente de fricción [4, alta dureza, buena resistencia al desgaste [5], entre otras. Ello hace que sean materiales sumamente interesantes para las aplicaciones en el desarrollo y producción tecnológica del país.

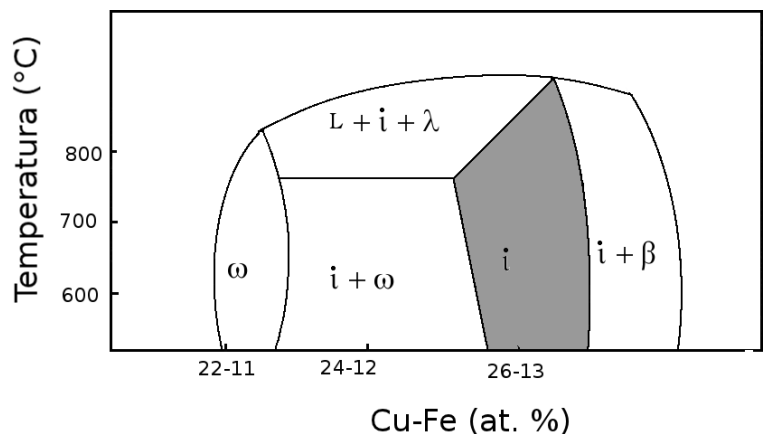

Figura 1: Diagrama de fases esquemático de la aleación $\mathrm{Al}-\mathrm{Cu}-\mathrm{Fe}$ que muestra las posibles fases a obtener al variar la temperatura $\left({ }^{\circ} \mathrm{C}\right)$ y el porcentaje de concentración de los elementos constituyentes (basado en las referencias [7, 8]).

\begin{tabular}{ccc}
\hline $\begin{array}{c}\text { Tiempo } \\
\text { (horas) }\end{array}$ & $\begin{array}{c}\text { Temperatura } \\
\left({ }^{\circ} \mathrm{C}\right)\end{array}$ & Referencia \\
\hline \multirow{2}{*}{6} & 600 & - \\
& 700 & - \\
& 800 & {$[9]$} \\
\hline \multirow{2}{*}{48} & 600 & - \\
& 700 & {$[10]$} \\
\hline \multirow{3}{*}{168} & 800 & - \\
& 600 & {$[11$} \\
& 700 & {$[12$} \\
\hline
\end{tabular}

Tabla 1: Parámetros empleados, la temperatura y el tiempo de tratamiento térmico, para la preparación de muestras cuasicristalinas en la composición $\mathrm{Al}_{64} \mathrm{Cu}_{23} \mathrm{Fe}_{13}$. Trabajos previos reportados para algunas condiciones específicas son indicados con su respectiva referencia.

Los primeros en reportar una fase icosaédrica estable, denominada fase- $i$, en el sistema de $\mathrm{AlCuFe}$ fueron A. P. Tsai y colaboradores [6], ellos encontraron que su formación era peritéctica y que su estabilidad resulta ser muy sensible a la temperatura y composición química. En el diagrama de fases del sistema $\mathrm{AlCuFe}$ mostrado en la figura 1 se puede observar que el área de la región icosaédrica, área sombreada, cambia con la variación de la temperatura y que en el límite de dicha región hay coexistencia con fases cristalinas $\lambda$ $\mathrm{Al}_{73} \mathrm{Cu}_{5} \mathrm{Fe}_{22}, \beta-\mathrm{Al}_{52} \mathrm{Cu}_{35} \mathrm{Fe}_{13}$ y $\omega-\mathrm{Al}_{70} \mathrm{Cu}_{20} \mathrm{Fe}_{10}$ [7, 8. Por lo tanto, es evidente que el proceso de tratamiento térmico tiene el fin de mejorar la pureza del material removiendo fases metaestables que puedan presentarse en coexistencia con la fase cuasicristalina y en consecuencia, mejorar sus propiedades físicas y/o químicas. Asimismo, se reporta en la literatura 9 12 que la fase cuasicristalina de $\mathrm{AlCuFe}$ puede obtenerse a distintas temperaturas y tiempos de tratamiento térmico.

Así, a pesar de la existencia de trabajos sobre la obtención del sistema $\mathrm{AlCuFe}$, es de interés realizar un estudio más sistemático de la síntesis y estabilidad de la fase- $i$; por ello en este trabajo se plantea preparar muestras cuasicristalinas por el método de horno de arco en la composición $\mathrm{Al}_{64} \mathrm{Cu}_{23} \mathrm{Fe}_{13}$ y luego someterlas a tratamiento térmico variando dos parámetros que son la temperatura y el tiempo, ver tabla 1, con el fin de estudiar su influencia en la estabilidad y formación de la aleación.

\section{Detalle experimental}

La síntesis del cuasicristal $\mathrm{Al}_{64} \mathrm{Cu}_{23} \mathrm{Fe}_{13}$ se realizó tomando como referencia el trabajo de M. Taquire [12, mezclando los elementos de alta pureza química como el $\mathrm{Al}(99.99 \%)$, el Cu (99.99\%) y el Fe (99.9\%)); posteriormente las mezclas fueron compactados con una prensa hidráulica manual para obtener la forma de pastillas cilíndricas. Estas pastillas fueron fundidas empleando la técnica de horno de arco en atmósfera controlada de argón para evitar la posible contaminación y/o oxidación de las muestras; el proceso de fundición se repitió dos veces con el fin de homogenizar la aleación, la cual finalmente tomó la forma de pequeñas esferas de $\sim 2 \mathrm{~cm}$ de diamétro.

Posteriormente las esferas fueron encapsuladas en tubos de cuarzo, también en atmósfera de argón, para someterlas a tratamiento térmico según las condiciones de temperatura y tiempo mostradas en la tabla 1, para un estudio más sistemático de la estabilidad del cuasicristal. La preparación de las muestras se llevó a cabo en dos etapas a las que llamaremos serie A y serie B, ésto con el fin de verificar la reproductibilidad de los resultados obtenidos.

Las muestras tratadas térmicamente fueron pulverizadas para su caracterización estructural por la técnica de difracción de rayos X (DRX) en un difractómetro Bruker D8 Focus con geometría Bragg Brentano usando una fuente radiactiva de $\mathrm{Cu}$ con longitud de onda $\lambda_{K \alpha 1}=1.5406 \AA$. Las intensidades difractadas fueron medidas en un intervalo $2 \theta$ de $20^{\circ}$ a $80^{\circ}$ con un paso de $0.04^{\circ}$ y un tiempo de 16 segundos por paso. La composición elemental de la muestra cuasicristalina fue analizada por espectroscopia de energía dispersiva de rayos $\mathrm{X}(\mathrm{EDX})$ en un microscopio óptico modelo FEI Quanta 200.

La morfología fue examinada por microscopía electrónica de barrido (MEB). 


\section{Resultados y discusión}

En la figura 2 se observa el difractograma de la muestra antes de ser sometida a tratamiento térmico, el cual fue comparado con los difractogramas de la base de datos JCPDS-ICDD donde se encontró además de la fase cuasicristalina con simetría icosaedral, denominada fase- $i$, una fase cristalina $\mathrm{Al}_{2} \mathrm{Cu}_{3}$, la cual es afín a la fase cuasicristalina. Por ello, con el propósito de remover ésta fase espuria necesariamente se someten las muestras cuasicristalinas a tratamiento térmico; para tal efecto empleamos los parámetros de tiempo y temperatura descritos en la tabla 1.

Los resultados obtenidos de las muestras tratadas térmicamente a diferentes tiempos y temperatura pertenecientes a la serie A se muestran en la figura 3, donde se puede apreciar que todos los difractogramas presentan sólo la fase cuasicristalina icosaedral, ya que los picos de difracción pueden ser indexados de acuerdo al modelo propuesto por Cornier-Quiquandon y colaboradores [13]. Tal como se observa en el primer difractograma de la figura 3(a) y al comparar las posiciones de los picos de Bragg de éste difractograma con los otros difractogramas de esta figura se encuentran que son similares, lo cual está en buen acuerdo con los resultados reportados en la literatura ya antes citada $69-12$. Asimismo, los difractogramas de las muestras preparadas en la serie B indican también sólo la presencia de la fase icosaedral, a excepción de dos muestras que fueron tratadas a $600{ }^{\circ} \mathrm{C}$ por 168 horas y $800{ }^{\circ} \mathrm{C}$ por 6 horas en las cuales se evidencia aún una fase cristalina $\mathrm{Al}_{4} \mathrm{Cu}_{9}$ remanente, tal como se muestra en la figura 4.

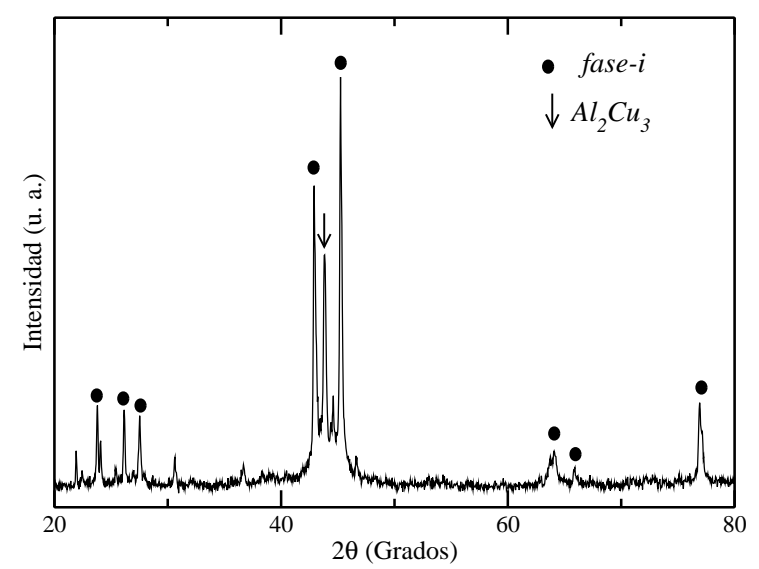

Figura 2: Difractograma de rayos $\mathrm{X}$ de la muestra cuasicristalina en coexistencia con la fase cristalina $\mathrm{Al}_{2} \mathrm{Cu}_{3}$.
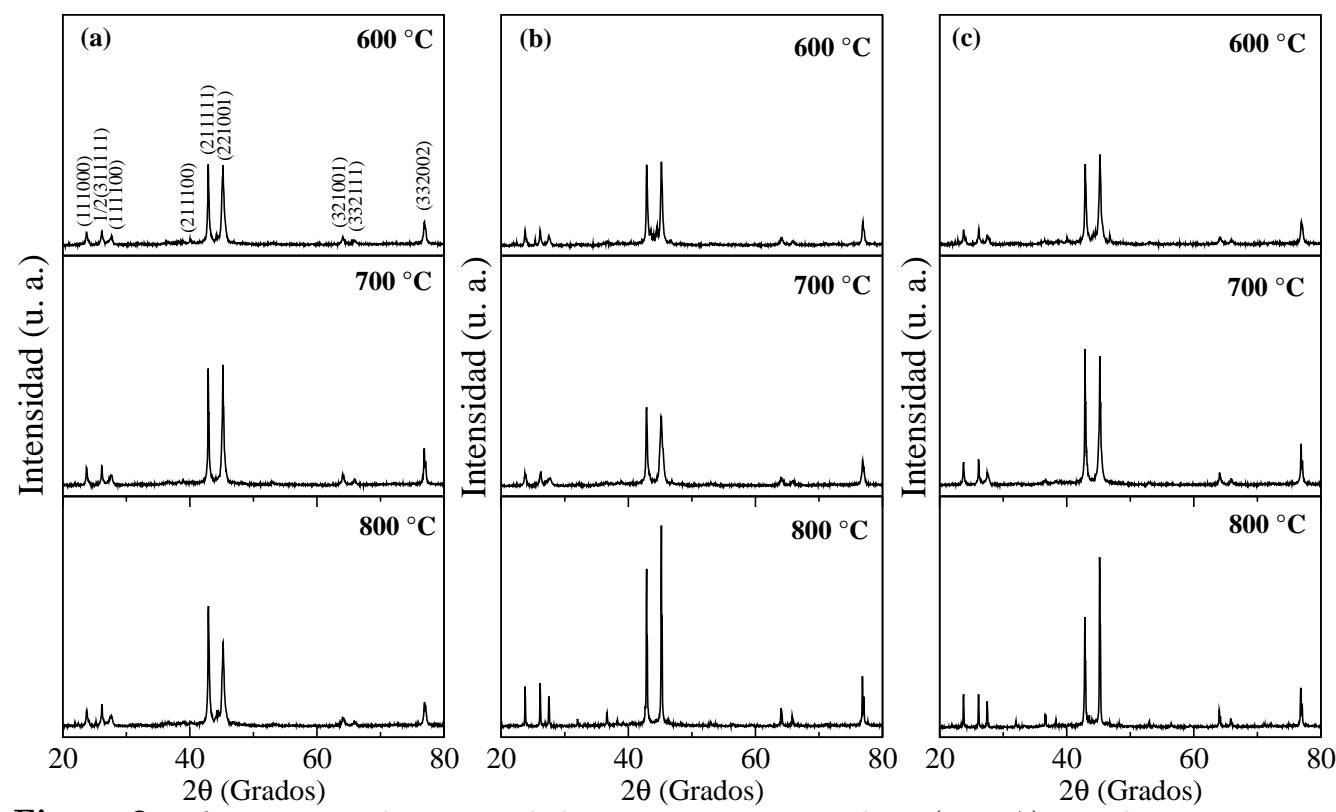

Figura 3: Difractogramas de rayos $X$ de las muestras cuasicristalinas (serie A) tratadas térmicamente a diferentes temperaturas por (a) 6 horas, (b) 48 horas y (c) 168 horas.

Analizando la figura 3, se observa que no todos los picos máximos de los difractogramas de la fase-i presentan las mismas intensidades; se observa que a la temperatura de $600{ }^{\circ} \mathrm{C}$ y tiempos de tratamiento térmico de 6, 48 y 168 horas las intensidades son pequeñas al igual que para $700{ }^{\circ} \mathrm{C}$ y $800{ }^{\circ} \mathrm{C}$ por 48 y 6 horas, respectivamente; mientras que para $700{ }^{\circ} \mathrm{C}$ durante $6 \mathrm{y}$ 168 horas hay un ligero incremento en las intensidades, 
más aún, a $800{ }^{\circ} \mathrm{C}$ por 48 y 168 horas, el incremento en la intensidad es mucho mayor. Estas variaciones en las intensidades de los picos, están relacionadas a la variación del ancho del pico a media altura y en conse- cuencia a la variación del tamaño de grano de acuerdo a la fórmula de Debye Scherrer aplicado al primer pico principal $\sim 42.8^{\circ}$. Los resultados obtenidos tanto de la serie A como de la serie B se muestran en la tabla 2.

\begin{tabular}{|c|c|c|c|c|}
\hline \multirow{2}{*}{$\begin{array}{l}\text { Tiempo } \\
\text { (horas) }\end{array}$} & \multirow{2}{*}{$\begin{array}{c}\text { Temperatura } \\
\left({ }^{\circ} \mathrm{C}\right)\end{array}$} & \multicolumn{3}{|c|}{ Tamaño de grano (nm) } \\
\hline & & Serie A & Serie B & Promedio \\
\hline \multirow{3}{*}{6} & 600 & 66 & 41 & 53.5 \\
\hline & 700 & 91 & 78 & 84.5 \\
\hline & 800 & 63 & $34^{*}$ & 48.5 \\
\hline \multirow{3}{*}{48} & 600 & 66 & 52 & 59.0 \\
\hline & 700 & 50 & 47 & 48.5 \\
\hline & 800 & 134 & 95 & 114.5 \\
\hline \multirow{3}{*}{168} & 600 & 47 & $34^{*}$ & 40.5 \\
\hline & 700 & 124 & 95 & 109.5 \\
\hline & 800 & 122 & 77 & 99.5 \\
\hline
\end{tabular}

Tabla 2: Tamaño de grano de las muestras cuasicristalinas de las series A y B tratadas térmicamente, donde el símbolo * corresponde a las muestras cuasicristalinas aún en coexistencia con una fase cristalina tal como se observa en la figura 4

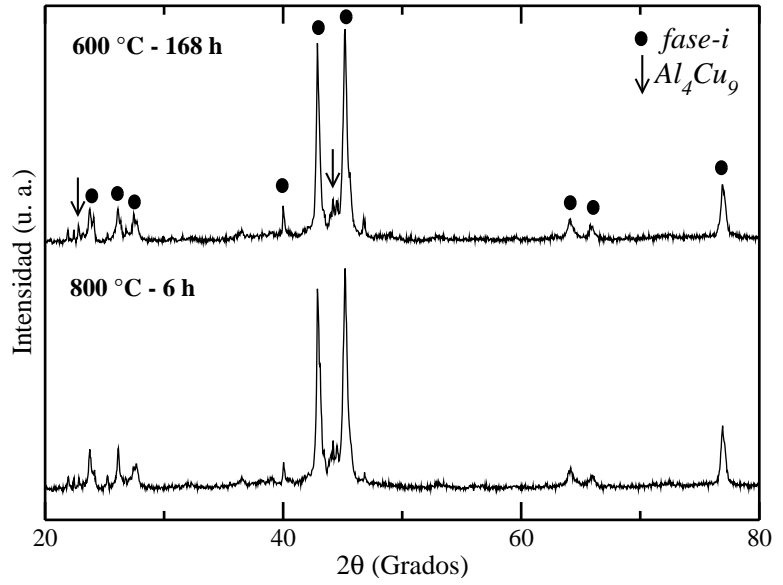

Figura 4: Difractogramas de rayos $\mathrm{X}$ de las muestras cuasicristalinas tratadas térmicamente donde aún se nota la presencia de una fase cristalina, $\mathrm{Al}_{4} \mathrm{Cu}_{9}$, remanente.

La figura 5(a) muestra las medidas MEB correspondientes a la región superficial de una muestra cuasicristalina antes de ser sometida a tratamiento térmico. De la morfología de la muestra se evidencia partículas de forma dodecaédrica que presenta la misma simetría que la del icosaedro, lo que es propio de sistemas cuasicristalinos correspondientes a la fase-i.

Además, se notan zonas con un color ligeramente oscurecido que podría corresponder a la fase cristalina mostrada en el análisis de difracción de rayos $\mathrm{X}$ de esta muestra en la figura 2. Por otro lado, en la figura 5(b), perteneciente a la sección transversal de la muestra cuasicristalina tratada térmicamente a $700{ }^{\circ} \mathrm{C} \mathrm{du}-$ rante 168 horas (serie A), observamos varios poliedros de morfología dodecaédrica y en mayor proporción que otras. Estos resultados están en concordancia con los resultados obtenidos por medio de difracción de rayos $\mathrm{X}$, mostrados en la figura 3 .

Asimismo, en la figura 6 se observa el análisis elemental por EDX de la muestra cuasicristalina tratada a $700{ }^{\circ} \mathrm{C}$ durante 168 horas, el cuadro superior insertado en la misma figura indica una ligera variación en la composición nominal ideal $\left(\mathrm{Al}_{64} \mathrm{Cu}_{23} \mathrm{Fe}_{13}\right)$ obteniéndose finalmente la composición $\mathrm{Al}_{65} \mathrm{Cu}_{22} \mathrm{Fe}_{13}$ (sin considerar la contribución del oxígeno), lo cual está dentro del rango composicional permitido para las muestras cuasicristalinas del sistema $\mathrm{AlCuFe}$ [14].

\section{Conclusiones}

Como resultado del presente estudio se ha comprobado que es necesario tratar térmicamente las muestras sintetizadas para eliminar las fases espurias y obtener una fase cuasicristalina pura. Ello se puede llevar a cabo bajo las condiciones descritas en la tabla 2. Del presente estudio se ha determinado que los cuasicristales de mayor tamaño de grano (por encima del promedio) son aquellos sometidos a tratamientos térmicos de $800{ }^{\circ} \mathrm{C}$ durante 48 horas o $700{ }^{\circ} \mathrm{C}$ durante 168 horas. Siguiendo este procedimiento se ha obtenido cuasicristales con granos cuyo tamaño se encuentra en el rango de 100 a $115 \mathrm{~nm}$. 


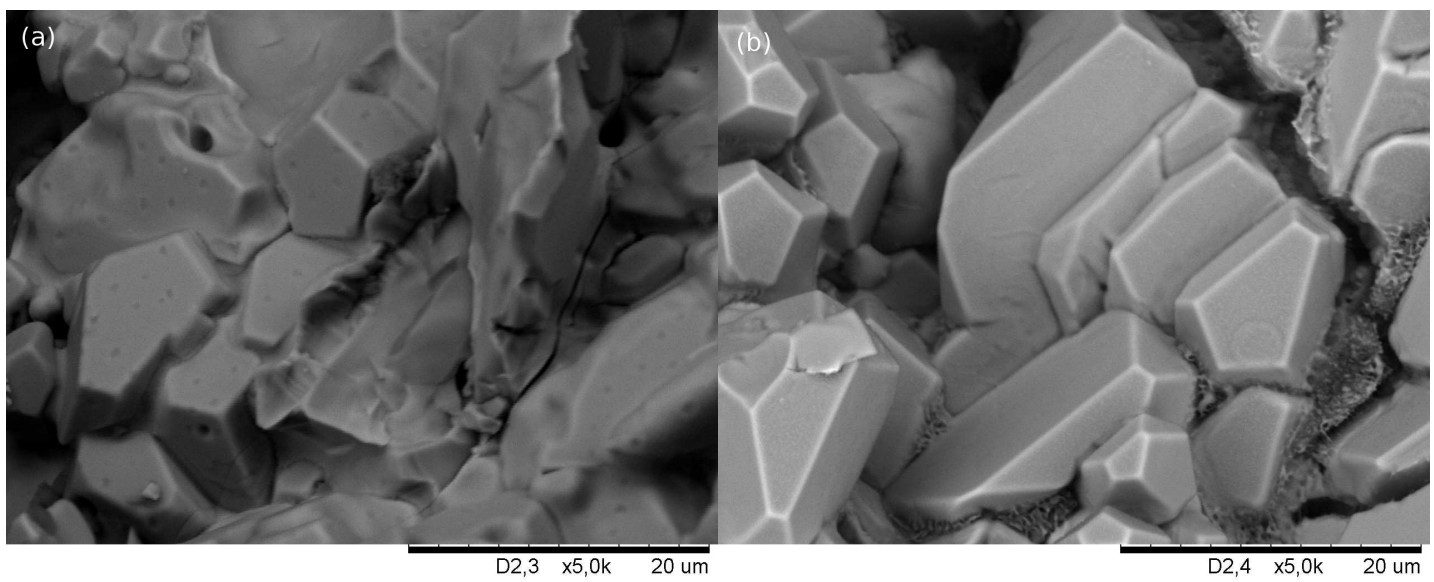

Figura 5: Micrografía por MEB de cuasicristales $\mathrm{Al}_{64} \mathrm{Cu}_{23} \mathrm{Fe}_{13}$ (a) Sin tratamiento térmico y (b) Tratada térmicamente a $700{ }^{\circ} \mathrm{C}$ durante 168 horas (serie A).

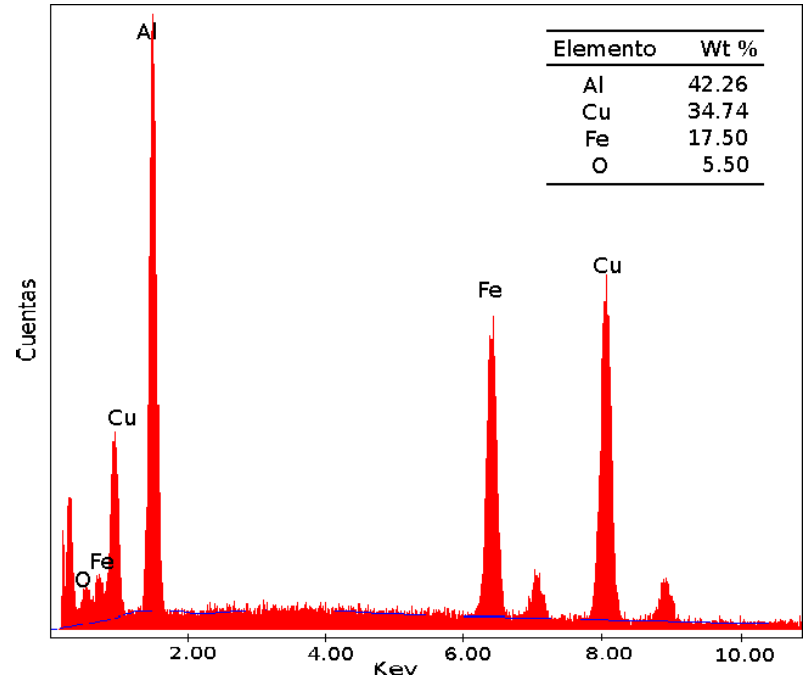

Figura 6: Análisis elemental por EDX de la muestra cuasicristalina tratada térmicamente a $700{ }^{\circ} \mathrm{C}$ durante 168 horas.

\section{Agradecimientos}

M. Z. P. y M. P. agradecen a la Asociación Familia Zuñiga y Rivero por el apoyo económico mediante el Programa de Becas para estudios de maestría en la
Facultad de Ciencias Físicas de la UNMSM . Asimismo, los autores agradecen al Vicerrectorado de Investigación de la UNMSM por el soporte financiero a través del Proyecto de Investigación Con-Con Nro. 111301011.

\section{Referencias}

[1] D. Levine y P. J. Steinhardt; Phys. Rev. Lett. 34, 596 (1986).

[2] D. Shechtman, I. Blech, D. Gratias y J. W. Cahn; Phys. Rev. Lett. 53, 1951 (1984).
[3] O. Rapp, Electronic Transport Properties of Quasicrystals Experimental Results. Physical Properties of Quasicrystals, Editado por Z. M. Stadnik, Springer-Verlag, Berlín (1998).

[4] J. M. Dubois, Mater. Sci. Eng. A 4, 294 (2000).

[5] D. J. Sordelet, M. F. Besser y J. L. Logsdon; Ma- 
ter. Sci. Eng. A 298, 284 (1998).

[6] A. Tsai, A. Inoue y T. Masumoto; J. Mater. Sci. Lett. 6, 194 (1987).

[7] Zbigniew M. Stadnik, Physical Properties of Quasicrystal, Springer, Berlín (1999).

[8] F. Faudot, A. Quivy, Y. Calvayrac, D. Gratias y M. Harmelin; Mater. Sci. Eng. A133, 338 (1991).

[9] A. P. Tsai y M. Yoshimura; Applied Catalysis A 214, 237 (2001).

[10] Z. M. Stadnik y G. Stroink; Phys. Rev. B, 39, 10447 (1988).
[11] N. K. Mukhipadyay, V. Kurup, V. C. Srivastava, P. Joshi y R. K. Mandal; J. Non-Cryst. Solid YY, 334 (2004).

[12] M. Taquire, Sintesis y caracterización estructural del cuasicristal nanoestructurado $\mathrm{Al}_{64} \mathrm{Cu}_{23} \mathrm{Fe}_{13}$, Tesis de Licenciatura, Universidad Nacional Mayor de San Marcos, Lima, Perú, (2009).

[13] M. Cornier-Quiquandon, A. Quivy, S. Lefebvre, E. Elkaim, G. Heger, A. Katz y D. Gratias; Phys. Rev. B 44, 2071 (1991).

[14] Elina Huttunen-Saarivirta, Journal of Alloys and Compounds, 363, 150 (2004). 